Iustitia Socialis. Revista Arbitrada de Ciencias Jurídicas.

Año IV. Vol. IV. N 1. Edición Especial 2019

Hecho el depósito de Ley: FA2016000064

ISSN: 2542-3371

FUNDACIÓN KOINONIA (F.K). Santa Ana de Coro, Venezuela

Yolange Véliz Valencia

http://dx.doi.org/10.35381/racji.v4i1.547

\title{
Perspectivas de la adopción homoparental en el Ecuador
}

Prospects for homoparental adoption in Ecuador

\author{
Yolange Véliz Valencia \\ nejaso2252@imail5.net \\ Universidad San Gregorio de Portoviejo, Manabí \\ Ecuador. \\ https://orcid.org/0000-0003-0862-3053
}

Recibido: 30 de octubre de 2019

Aprobado: 03 de diciembre de 2019

\begin{abstract}
RESUMEN
El artículo tiene como objetivo analizar las perspectivas de adopción homoparental en Ecuador. La relevancia del estudio está relacionada con la homoparentalidad, que se asocia con valores de una nueva era, diversa e inclusiva; desde la reconsideración de posiciones científicas que conjeturaban la homosexualidad como una enfermedad o perversión en el debate académico legal, a pesar del reconocimiento de las diferentes tipos de familias. Se trabajó metodológicamente con una investigación documentalbibliográfica bajo las técnicas de análisis juridico, a partir de documentos y casos relacionados con el tema planteado. Los resultados permiten enfocar todos estos aspectos desde los valores axiológicos del Ecuador, planteando la dignidad humana como un bien superior en garantía constitucional.
\end{abstract}

Descriptores: Adopción; bienestar de la infancia; derechos del niño; discriminación.

\begin{abstract}
The article aims to analyze the prospects for homoparental adoption in Ecuador. The relevance of the study is related to homoparentality, which is associated with values of a new era, diverse and inclusive; from the reconsideration of scientific positions that conjectured homosexuality as a disease or perversion in the legal academic debate, despite the recognition of different types of families. We worked methodologically with a documentary-bibliographic investigation under the techniques of legal analysis, based on documents and cases related to the topic raised. The results allow to focus all these aspects from the axiological values of Ecuador, stating human dignity as a superior good in guarantee constitutes.
\end{abstract}


Descriptors: Adoption; child welfare; rights of the child; discrimination.

\section{INTRODUCCIÓN}

La adopción, como institución jurídica, es otra variable que termina por complejizar los distintos enfoques que se han desarrollado en esta materia, estimulando inquietudes e interrogantes que giran en torno a los valores tradicionales y al concepto judeocristiano de familia, que aún hoy día subyace. En el año 2016 se presentó en la Organización de Naciones Unidas (ONU) una resolución del Consejo de Derechos Humanos de las Naciones Unidas para la creación de una Relatoría Especial de "Protección contra la violencia y la discriminación basada en la orientación sexual y la identidad de género", 18 países votaron en contra y se produjeron 6 abstenciones, que suman más de los 23 votos a favor, con lo cual se instituye la complejidad de la materia.

En este orden de ideas, la homoparentalidad es una figura que se inserta en la realidad jurídica y sobre ella surgen interesantes debates en numerosos países. Asimismo, son varias las instituciones que respaldan los esfuerzos de estos sectores y comunidades por su visibilización y adecuado reconocimiento, en especial, al momento de forjar una familia, entre ellas la Federación Española de Sociedades de Sexología, concuerda que la homosexualidad por sí sola no debe ser un explicación válida como norma de prohibición para que parejas del mismo género adopten, toda vez que diversas investigaciones antropológicas realizadas a través de las culturas y los tiempos que versan sobre familias, hogares y las relaciones que de ellas surgen, no proporcionan apoyo alguno a la idea de que la civilización o un orden social viable dependen de la familia como una institución únicamente heterosexual. (Federación Española de Sociedades de Sexología, 2005).

En este contexto, es menester subrayar que la colonización española en los pueblos latinoamericanos, no solo impuso reyes y políticas sino también una estructura moral basada en la religión que profesaba el conquistador, creencia que ha permanecido (intocada) hasta nuestros días. Por su parte, Chaparro Piedrahíta \& Guzmán Muñoz 
(2017), sostiene que la adopción homoparental representa un cambio paradigmático desde el contexto social arraigado tradicionalmente.

En el contexto social local del Ecuador, encontramos algunos avances en esta materia, que se incorporan por la voluntad del constituyente en el texto fundamental del año 2008, convirtiendo al país, en una nación garantista de los derechos de la Comunidad de Lesbianas, Gays, Bisexuales, Transexuales, Transgéneros e Intersexuales, conforme los estipulado en el artículo 11 que consagra que nadie podrá ser discriminado por razones de sexo, identidad de género u orientación sexual.

Ahora bien, la Constitución de Ecuador del año 2008, señala textualmente en el artículo 67 que: "el matrimonio es la unión entre hombre y mujer" y que "la adopción corresponderá solo a parejas de distinto sexo". Paradójicamente, la misma norma reconoce: "la familia en sus diversos tipos", así como la "unión monogámica". Teniendo en cuenta los argumentos que grosso modo se han perfilado, nos preguntamos: ¿La Constitución de la República del Ecuador al impedir la adopción homoparental es coherente con los principios que le sirven de fundamento para su aplicación?

Para dar respuesta a esta incógnita se trazó como objetivo general analizar las perspectivas de la adopción homoparental en el Ecuador, siguiendo el análisis de algunas vertientes científico - jurídicas que se relacionan con el propósito descrito.

\section{MÉTODO}

La investigación fue de tipo documental-bibliográfico bajo las técnicas del análisis jurídico, Palella Stracuzzi y Martins Pestana (2012), comentan que esta investigación permite al investigador generar un análisis reflexivo sobre un tema, con base a investigaciones, documentos, leyes, jurisprudencias, relacionadas con la variable de estudio, constituyéndose además, en la población a escrutar. Así mismo, los autores plantean que el diseño bibliográfico permite realizar una indagación sistematizada de los elementos involucrados en el proceso investigativo, aplicándose para tal fin la técnica de análisis de contenido jurídico con la finalidad de destacar los aspectos más relevantes presentes en el manuscrito objeto de muestra poblacional. 


\section{RESULTADOS}

\section{La Familia en la Constitución de la República del Ecuador.}

La familia es el elemento activo. Nunca permanece estacionada, sino que pasa de una forma inferior a otra superior a medida que la sociedad evoluciona de un grado más bajo a otro más alto. Los sistemas de parentesco, por el contrario, son pasivos, sólo después de largos intervalos registran los progresos hechos por la familia y sólo sufren una modificación radical cuando la familia se ha modificado radicalmente. (Engels, 1884)

Este planteamiento de Federico Engels en el año de 1884, fue la primera aproximación a la familia como fenómeno dialéctico en la sociedad, argumento que encuentra su desarrollo por numerosos autores contemporáneos. La Constitución de la República del Ecuador de 2008, parece haber reconocido este planteamiento, cuando el artículo 67 enuncia:

Se reconoce la familia en sus diversos tipos. El Estado la protegerá como núcleo fundamental de la sociedad y garantizará condiciones que favorezcan integralmente la consecución de sus fines. Estas se constituirán por vínculos jurídicos o de hecho y se basarán en la igualdad de derechos y oportunidades de sus integrantes".

Destaca de la norma el primer párrafo que obliga a repensar el concepto tradicional acerca de la familia, para reconocer la diversa tipología que puede estar vinculada con el sustantivo "Familia". Por su parte, el artículo 68 guarda plena concordancia con el segundo aparte del artículo 67, al señalar que el matrimonio es el acto jurídico entre un hombre y una mujer, reiterando este aspecto al prescribir que:

"La unión estable y monogámica entre dos personas libres de vínculo matrimonial que formen un hogar de hecho, por el lapso y bajo las condiciones y circunstancias que señale la ley, generará los mismos derechos y obligaciones que tienen las familias constituidas mediante matrimonio.

Finalmente, la disposición que antecede termina por señalar que la adopción "corresponderá sólo a parejas de distinto sexo", con lo cual a primera vista se revelan claras contradicciones. No existe diafanidad en el texto fundamental sobre las 
enunciadas instituciones jurídicas, ni se sigue una sola línea de pensamiento, puesto que el reconocimiento de las diversas tipologías de familia, colisiona con la definición de la institución del matrimonio, acto que se regula únicamente sobre personas de sexos distintos y la adopción está circunscrita a tal instancia, suerte de la que no escapa la vigencia de la unión de hecho que a la sazón de sociedad de gananciales cumplen los mismos efectos. Desde todas las aristas, se colige una ambigüedad normativa, que se magnifica, al proscribir el matrimonio de las parejas homosexuales; sin embargo, si se reconoce la capacidad de establecer la unión de hecho reconocida legalmente y con iguales efectos.

\section{La comunidad LGTBIQ+}

Como lo señaláramos, la complejidad del tema pasa por el reconocimiento de la igualdad ante la ley, y la prohibición de cualquier forma de discriminación que instituye la Constitución de la República del Ecuador en el artículo 11 como principio de aplicación, el cual se consustancia con lo dispuesto en el artículo 10, que reconoce a las personas, comunidades y colectivos como titulares de derechos. En este particular se inscriben las comunidades LGTBIQ+, quienes son actores fundamentales de las conquistas ciudadanas en favor del respeto de los derechos y garantías de las personas que las conforman.

En contraste con esta concepción, es relevante que recordemos que la Medicina y la Psiquiatría se dedicaron al estudio de la sexualidad, y aun cuando sus argumentos contradecían los estudios de otras ciencias, forjaron distintas concepciones que hasta finales del siglo XX asentaron su atención en consideraciones sobre la orientación sexual y su relación con distintas enfermedades y aberraciones.

El feminismo y los movimientos sociales por la liberación homosexual indujeron a la revisión del arcaísmo de los aludidos argumentos, en efecto, las luchas del Movimiento Gay surgido en los Estados Unidos a partir del 1969, incidieron en la decisión que tomó la Asociación Americana de Psiquiatría en el año 1973 de retirar la homosexualidad de su lista de trastornos mentales contemplada en el Manual Diagnóstico y Estadístico de 
Ios Trastornos Mentales DSM III (Diagnostic and Statistical Manual of Mental Disorders DSM). Diecisiete años después de que la Asociación Americana de Psiquiatría retirara la homosexualidad de su Manual de Trastornos y la Organización Mundial de Salud (OMS) también resolvió eliminar la homosexualidad de su Manual de Clasificación Internacional de Enfermedades (CIE).

Aunque la OMS tomó la decisión de excluir la homosexualidad de su lista de enfermedades mentales, no ocurrió lo mismo con la transexualidad. Hasta la actualidad, el CIE X concibe la transexualidad como patología. Por su parte, no fue hasta el 2013 que en la versión DSM V se eliminó la noción de "trastorno de identidad de género" que consideraba a la transexualidad como enfermedad mental. Lo cierto es que, las transformaciones del pensamiento científico sobre la sexualidad han influido en las nuevas concepciones acerca las personas que conforman la comunidad LGBTIQ+.

Lo expuesto nos lleva a la exploración del término diversidad sexual que precisa "la idea de que las personas tienen existencias sexuales, de género y eróticas diversas, que son parte de una realidad humana que es necesario respetar" (Núñez Noriega, 2011), de allí se funda el valor de la dignidad humana.

Entendemos que, los seres humanos poseen realidades de género que van más allá de lo establecido por el sistema binario sexo-género, a saber: hombre-masculino/mujerfemenina. Las posibilidades de construir una identidad de género distinta a la que la sociedad asigna a una mujer o un hombre al nacer son variadas, y en este sentido la existencia de un hombre femenino o una mujer masculina, es parte de la diversidad sexo-genérica de las personas. (Butler, 2006)

Ahora bien, si alguien es considerado por su condición sexual un ser inferior, anormal y al margen de la ley, entonces se presenta un grave problema al momento de reconocer a esa persona en su dignidad, por lo que uno de los primeros pasos del derecho internacional moderno para definir la condición de igualdad de todos los seres humanos fue promulgar en 1948 la Declaración Universal de Derechos Humanos, la misma que consagra en su artículo 2: "Toda persona tiene todos los derechos y libertades proclamados en esta Declaración, sin distinción alguna de raza, color, sexo, idioma, 
religión, opinión política o de cualquier otra índole, origen nacional o social, posición económica, nacimiento o cualquier otra condición".

En el Ecuador, este tema ha sido ampliamente debatido. Incluso ha llamado la atención de altas personalidades de la política nacional, a título ilustrativo resaltamos que el presidente Rafael Correa propuso una consulta popular en las elecciones seccionales de febrero de 2014 para que los ecuatorianos decidieran la legalización de las bodas gays. En febrero de 2013 se generó una polémica similar. El ex candidato presidencial Nelson Zavala sostuvo en su campaña que "Dios creó a Adán y Eva, no a Adán y Esteban" y agregó que "la Biblia es clara: ni los afeminados ni los que se echan con otros varones heredarán el reino de Dios". Tras esas declaraciones, activistas LGBTIQ+ lo demandaron y en marzo de 2013 el Tribunal Contencioso Electoral (TCE) condenó al pastor evangélico a pagar una multa de diez salarios mínimos (USD 3.180) y también perdió sus derechos políticos por un año. Sus expresiones fueron consideradas homofóbicas.

De hecho, el Consejo Nacional Electoral prohíbe "la emisión pública de cualquier expresión que discrimine o afecte a la dignidad de las personas, o utilicen símbolos, expresiones de carácter religioso". La discusión sobre estos temas discurre en la necesidad de operativizar los derechos contenidos en la Constitución de la República del Ecuador, ya que la invisibilidad de estos afecta de manera directa la dignidad humana de todas las personas que conforman la comunidad LGBTQ+, y atenta contra la igualdad de trato y de oportunidades, obstaculizando el acceso al empleo, la vivienda, los servicios públicos, las pensiones, los beneficios de salud, etc. (Movimiento Nacional LGBTI, 2013).

Todos estos planteamientos han sido observados por el Estado, quien dentro del Plan Nacional de Buen Vivir 2009-2013, emitido por la Secretaria Nacional de Planificación y Desarrollo, procura la garantía de la igualdad para las personas LGBTIQ+. El Primer Objetivo Nacional para el Buen Vivir, es precisamente: "Auspiciar la igualdad, cohesión e integración social y territorial en la diversidad", y en su política 1.6 consta textualmente "Reconocer y respetar las diversidades socioculturales y erradicar toda 
forma de discriminación, sea ésta por motivos de género, de opción sexual, étnicoculturales, políticos, económicos, religiosos, de origen, migratorios, geográficos, etarios, de condición socioeconómica, condición de discapacidad u otros".

También en el capítulo 3 se dispone: "Un Cambio de Paradigma: del Desarrollo al Buen Vivir", en su punto 3.2.10. "Hacia un Estado democrático, pluralista y laico", prescribe el ejercicio de los derechos sexuales y los derechos reproductivos, y cómo estos son de carácter laico, enfatizando que el Estado es el responsable de garantizar el respeto de las opciones autónomas de cada individuo. Si bien es cierto, el artículo 68 de la Constitución reconoce la unión de hecho entre parejas del mismo sexo, y lo hace reconociendo los mismos derechos y obligaciones que tiene el matrimonio, termina por exceptuar la adopción. Del mismo modo, es relevante recordar que este reconocimiento no es considerado como un estado civil.

Conforme con lo señalado tenemos que, a pesar de las varias menciones que se hace al derecho a la igualdad y a la no discriminación, también la Constitución ha puesto trabas en el ejercicio de una igualdad real para las personas pertenecientes a esta comunidad diversa, el ejemplo del no reconocimiento del matrimonio civil igualitario y la adopción para parejas del mismo sexo, es restrictivo de derechos y discriminatorio para las parejas LGBTIQ+. El principal argumento para el no reconocimiento de estos derechos es que existe la Unión de Hecho, que se supone daría los mismos derechos que un matrimonio, sin embargo, no es un estado civil y la práctica ha demostrado que esta aparente igualdad que consagra la Constitución no se aplica. (Informe sobre la situación de los Derechos Humanos de las poblaciones LGBTI, 2013).

\section{La Adopción y los derechos del niño}

Un tema de gran complejidad se presenta respecto de la adopción por parte de familias homoparentales, ya que involucra toda la concepción que nace del paradigma de la Protección Integral, que acoge la República del Ecuador luego de la suscripción de la Convención Internacional sobre los Derechos del Niño de 1989, que relieva la vigencia de principios ampliamente descritos en esta norma, con ocasión de los instrumentos 
internacionales que fueran redactados y promulgados desde 1959. Estos principios dan cuenta de la necesidad de que el niño goce de una protección especial, con lo cual los Estados, procuraran dotar:

...de oportunidades y servicios, dispensado todo ello por la ley y por otros medios, para que pueda desarrollarse física, mental, moral, espiritual y socialmente en forma saludable y normal, así como en condiciones de libertad y dignidad. Al promulgar leyes con este fin, la consideración fundamental a que se atenderá será el interés superior del niño (UNICEF, 1959, Principio 2).

El interés superior del niño es el eje transversal sobre el cual se edifica toda acción en esta materia, el mismo sirve como parámetro de actuación, y precisamente ha sido este el nudo gordiano a la hora de interpretar el sentido de las normas que regulan la adopción como institución jurídica. La Convención Internacional sobre los Derechos del Niño de 1989, es muy clara en sus propósitos, pero el conflicto se genera cuando la moral de la sociedad se impone en temas tan complejos como el tratado. Las posturas dominantes en esta materia, se han reconocido como discriminatorias o excluyentes de la diversidad, impidiendo la inclusividad de las familias homoparentales alegando que se atenta contra el desarrollo saludable del niño o niña respecto de la crianza en un medio que es considerado amoral.

\section{La religión y sus posiciones con respecto a los derechos de la comunidad LGBTI en Ecuador}

Otra de las perspectivas que se suma a las consideraciones explanadas, se ubica bajo el tema religioso. En el censo realizado por la INEC en el año 2012:

En lo que se refiere a la filiación religiosa, los datos demuestran que el $91,95 \%$ de la población afirma tener una religión, de los cuales el $80,4 \%$ pertenece a la religión Católica, el 11,3\% Evangélica, 1,29\% Testigos de Jehová y el restante $6,96 \%$ pertenecen a otras religiones. (INEC, 2012)

En franca concordancia con lo expuesto, la comunidad religiosa en octubre de 2017, inició la campaña "Con mis hijos no te metas", en esta oportunidad, los LGBTIQ+ llegaron con una gran bandera del arcoíris y pancartas. "No son profamilia, son 
antiderechos". La importancia de la confrontación, es que las demandas ciudadanas llegaron hasta un tribunal de justicia. Precisamente, los activistas de la comunidad LGBTIQ+ demandaron a la Conferencia Episcopal Ecuatoriana (CCE) y al movimiento Vida y Familia, acusándolos de generar un ambiente de hostilidad y odio contra su colectivo. Aseguran que la campaña 'Con mis hijos no te metas' y la marcha que se realizó el pasado sábado ponen en riesgo el respeto de la diversidad y de las minorías sexuales. Mencionaron incluso haber recibido mensajes amenazantes, sobre todo por las redes sociales. Ellos culpan, directamente, a los denominados grupos profamilia. (Gaibor, 2017)

Enfatizamos en el hecho de que la marcha fue convocada para rechazar reformas a la Ley de Educación y el Proyecto de Ley contra la Violencia a la Mujer y, en efecto, hubo declaraciones dignas de censura de ciertos actores de la Iglesia, ya que estas manifestaciones atentan contra la dignidad humana y la igualdad ante ley, valores que impiden tratos discriminatorios contra las personas. El obispo auxiliar de Quito, monseñor Danilo Echeverría, manifestó que, pese a que la convocatoria ha partido de la sociedad civil, la Iglesia se ha solidarizado.

De igual forma destacamos el hecho de que la Iglesia ha expresado públicamente su oposición al matrimonio de personas del mismo sexo, y si relacionamos el dato previamente reseñado, acerca de que el Ecuador es un país donde el $80 \%$ de la población es católica, es fácil colegir los desafíos que afronta esta comunidad, para nadie es un secreto que la Iglesia ha liderado la postura moral de los pueblos de la América, por lo que, al tomar partido en contra del matrimonio entre personas del mismo sexo y la adopción homoparental, induce actitudes de intolerancia y discriminación.

En este escenario, manifestaciones de esta naturaleza, amén de ser una muestra de la libertad de expresión de los grupos que reclaman su derecho a orientar a sus hijos en los valores que ellos consideran morales, también hace explícita la intolerancia de un amplio sector de la población. 


\section{Perspectiva sobre la adopción homoparental}

Finalmente, la adopción homoparental parece ser un ideal utópico en el Ecuador, basta con señalar el gran debate que vivió el Ecuador, precisando que el 2 de abril de 2016, la atención se centró entre aprobar o no la adopción de menores por parte de parejas del mismo sexo, después de que una pareja de lesbianas inglesas interpusiera una apelación ante la Corte Constitucional buscando registrar a una menor de edad como hija de ambas. La pareja de lesbianas residente en Ecuador ha buscado registrar con sus dos apellidos a la niña, nacida en diciembre de 2011 tras la inseminación artificial de una de las mujeres.

Buil (2004) afirma que uno de los factores que más obstruye la aceptación de la adopción por parte de parejas de igual sexo es la resistencia al cambio que padece un amplio segmento de la sociedad lo cual genera temor, presupone alteración psíquica en el niño o niña y le estigmatiza anticipadamente ante su diferente situación familiar. Es válido admitir que, aun cuando no hay datos científicos sobre lo argumentado, dichos presupuestos se conservan en casi todos de los sectores sociales, además permean las aristas del poder encargado de decidir en la regulación de este tema.

Sin embargo, la falta de regulación de la adopción por parte de parejas del mismo sexo, en el país y en la comunidad internacional no ha impedido que sea una realidad que hoy muchos menores hagan parte de familias homoparentales, y que por el debate retórico moral y la falta de leyes que regulen la situación permanecen legalmente desprotegidos. Los diversos estudios en las áreas de la sociología y la psicología sobre la adopción de menores de edad por parejas del mismo sexo, no lograron demostrar daños para el desarrollo emocional, psicológico y sexual de los menores que habitan en hogares constituidos por familias homoparentales, aun cuando los acérrimos detractores han planteado que estos estudios están sesgados.

Navarro (2013) rescata tanto las posiciones en contra como las a favor entorno a la adopción homoparental, destacando las posturas en contra están basadas en argumentos tradicionales con una fuerte carga moral, ilustra en este sentido, lo referido por la catedrática de la Universidad Pontificia Bolivariana, María Eugenia Agudelo 
Bedoya, quien afirma que: "la institución de la familia está conformada por personas con roles tradicionales, padres con funciones económicas y autoridad, y madres con funciones protectoras y reproductivas"(Navarro, 2013), con lo cual queda evidenciado una postura retrógrada de los roles que hoy día corresponde desempeñar a los miembros de una familia.

En esta misma línea se inscribe Anatrella (2011), quien indica que los estudios realizados sobre los niños y niñas que han crecido en familias homoparentales, según los cuales no se ha detectado alteraciones psíquicas ni sociales en los menores como posible causa de vivir con padres de igual sexo, no evidencian la realidad, sino que intentan legitimar un cambio social a base de artificios, en dicho estudio el autor se centra en el atisbo antropológico examinado por pocos en este asunto. Considera que aceptar que personas de igual sexo conformen una familia que incluya hijos, ya sea adoptados o concebidos a través de la utilización de medios de asistencia médica, es una amenaza al derecho que tienen los niños a nacer y vivir en una familia constituida por una mujer y un hombre; pues a estas criaturas nadie les preguntará si desean o no pertenecer a una familia de situación particular (por ser contrario al concepto tradicional de familia), sobreponiendo entonces las aspiraciones afectivas de los adultos ante las necesidades y derechos de los niños a partir de los cuales debería definirse la filiación. Inferimos que indistintamente de los argumentos planteados, el núcleo esencial del problema estriba en la dignidad humana, que sirve de dique de contención para evitar cualquier clase de trato discriminatorio al que puedan quedar sometido las personas, que acorde con lo dispuesto en el artículo 10 de la Constitución, son titulares de derechos, en este caso nos referimos a los adoptantes del mismo sexo que conforman la diversidad de familias que hoy si encuentran asidero en el artículo 67 eiusdem, Por otra parte, es un deber impretermitible del Estado velar por el interés superior del niño, niña o adolescente, al permitir la adopción como institución de gran relevancia jurídico-social, en el sentido que se está ante un paradigma que reconoce la protección integral y la constitución de familias diferente a las convencionalmente aceptadas por la sociedad. 


\section{CONCLUSIONES}

La necesidad de esta reflexión se sustenta en el hecho de que, a pesar de que en Ecuador la norma constitucional protege a la familia en sus diversos tipos, no ha sido reformada la ley ordinaria, en correspondencia con el aludido avance, es por esta razón que, durante los últimos años la Federación Ecuatoriana de Organizaciones LGBTIQ+ en Ecuador se ha hecho presente en todos los ámbitos sociales reclamando el derecho constitucional a la igualdad y la no discriminación amparados en el fallo de la Corte Interamericana de Derechos Humanos (Corte IHD) que insta a los países del continente a reconocer el matrimonio entre personas del mismo sexo con plenos derechos.

Resaltamos como dato de interés las experiencias de otras latitudes, en este caso, países como España y Estados Unidos han legalizado la adopción homoparental, atendiendo a los cambios de paradigmas advertidos.

Del mismo modo, hay que reconocer lo dispuesto por la Corte IHD, quien ha destacado lo siguiente: "en sociedades democráticas debe existir coexistencia mutuamente pacífica entre lo secular y lo religioso", sin que una esfera interfiera en la otra, dicha resolución busca realzar el valor de la dignidad humana, y el principio de igualdad ante la ley, que, en nuestro ámbito jurídico, se convierte en un principio de interpretación y aplicación del orden jurídico.

Ante lo expuesto, la exigencia principal pasa por la consideración de un acertado diagnóstico de todas las aristas que involucra este espinoso tema, atendiendo las necesidades humanas y sociales, que a la postre terminará por justificar o no la necesidad de un cambio legislativo en cuanto al reconocimiento de la diversidad y de los derechos de las minorías, que están conformadas por personas dignas e iguales. Pero también la comunidad científica puede aportar en este contexto herramientas de gran valor para ofrecer respuestas racionales a las demandas ciudadanas, mediante la evaluación de aspectos sociales, psicológicos, morales, jurídicos, entre otros, que den la pauta para iniciar un diálogo asertivo en temas tan sensibles como los expuestos, basta por señalar que la ausencia de regulación no impide que esta realidad se 
materialice, y que la adopción no es un obstáculo para la crianza de niños, niñas y adolescentes por parejas del mismo sexo.

\section{REFERENCIAS CONSULTADAS}

1. Anatrella, T. (2011). La adopción de niños por personas homosexuales. Humanitas: revista de antropología y cultura cristiana, 80-93.

2. Butler, J. (2006). Deshacer el género. Buenos Aires: Paidós.

3. Chaparro Piedrahíta, Laura Juliana, \& Guzmán Muñoz, Yudy Marcela. (2017). Adopción homoparental: Estudio de derecho comparado a partir de las perspectivas de los países latinoamericanos que la han aprobado. Revista CES Derecho, 8(2), 267-297. recuperado de http://www.scielo.org.co/scielo.php?script=sci_arttext\&pid=S2145$77192017000200005 \&$ Ing=en\&tlng=es.

4. Engels, F. (1884). El Origen de la Familia, la propiedad privada y el estado. (Moscú, Ed.) Hottingen-Zürich: Progreso.

5. Gaibor, I. (2017). Los obispos evitan la cita con el juez por el conflicto de género. Quito, Pichincha, Ecuador. Recuperado de http://www.expreso.ec/actualidad/losobispos-evitan-la-cita-con-el-juez-por-el-conflicto-de-genero-XM1781189

6. INEC, E. (2012). INEC presenta por primera vez estadísticas sobre religión. Quito, Pichincha, Ecuador. Recuperado de http://www.ecuadorencifras.gob.ec/inec-presenta-por-primera-vez-estadisticassobre-religion/

7. Fundación Ecuatoriana Equidad. (2013). Informe sobre la situación de los Derechos Humanos de las poblaciones LGBTI. recuperado de https://biblio.flacsoandes.edu.ec/libros/digital/54809.pdf

8. Navarro, L. R. (2013). Posiciones en contra y en favor de la adopción homoparental desde la sociología y la psicología. Revista de Derecho Privado, 185-218. Recuperado de https://revistas.juridicas.unam.mx/index.php/derechoprivado/article/view/9022/11072

9. Núñez Noriega, G. (2011) ¿Qué es la diversidad sexual? Reflexiones desde la academia y el movimiento ciudadano. CIAD Centro de Investigación en Alimentación y Desarrollo. Quito: Abya-Yala. 
10. Palella Stracuzzi, S. \& Martins Pestana, F. (2012). Metodología de la investigación cuantitativa. Fondo editorial de la Universidad Pedagógica Libertador. Caracas, Venezuela.

11. UNICEF, O. (1959). Declaración Universal de los Derechos del Niño. ONU, Asamblea General. recuperado http://www.un.org/es/comun/docs/?symbol=A/RES/1386(XIV).

\section{REFERENCES CONSULTED}

1. Anatrella, T. (2011). The adoption of children by gay people. Humanitas: magazine of anthropology and Christian culture, 80-93.

2. Butler, J. (2006). Undo the gender. Buenos Aires: Paidós.

3. Chaparro Piedrahíta, Laura Juliana, \& Guzmán Muñoz, Yudy Marcela. (2017). Homoparental adoption: Law study compared from the perspectives of the Latin American countries that have approved it. CES Law Magazine, 8 (2), 267-297. Retrieved from http://www.scielo.org.co/scielo.php?script=sci arttext\&pid=S2145$\underline{77192017000200005 \& \operatorname{lng}=\text { en\&t|ng=en. }}$

4. Engels, F. (1884). The Origin of the Family, private property and the state. (Moscow, Ed.) Hottingen-Zürich: Progress.

5. Gaibor, I. (2017). The bishops avoid the appointment with the judge for the gender conflict. Quito, Pichincha, Ecuador. Recovered from http://www.expreso.ec/actualidad/los-obispos-evitan-la-cita-con-el-juez-por-elconflicto-de-genero-XM1781189

6. INEC, E. (2012). INEC presents statistics on religion for the first time. Quito, Pichincha, Ecuador. Recovered from http://www.ectadorencifras.gob.ec/inecpresenta-por-primera-vez-estadisticas-sobre-religion/

7. Ecuadorian Equity Foundation. (2013). Report on the Human Rights situation of LGBTI populations. retrieved from https://biblio.flacsoandes.edu.ec/libros/digital/54809.pdf

8. Navarro, L. R. (2013). Positions against and in favor of homoparental adoption from sociology and psychology. Private Law Magazine, 185-218. Recovered from https://revistas.juridicas.unam.mx/index.php/derechoprivado/article/view/9022/11072 


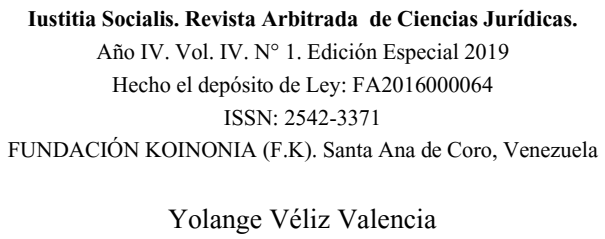

Yolange Véliz Valencia

9. Núñez Noriega, G. (2011) What is sexual diversity? Reflections from the academy and the citizen movement. CIAD Center for Research in Food and Development. Quito: Abya-Yala.

10.Palella Stracuzzi, S. \& Martins Pestana, F. (2012). Quantitative research methodology. Editorial Fund of the Libertador Pedagogical University. Caracas Venezuela.

11. UNICEF, O. (1959). Universal Declaration of the Rights of the Child. UN, General Assembly. Retrieved http://www.un.org/en/comun/docs/?symbol=A/RES/1386(XIV). 